\title{
Screening e dose-resposta de herbicidas à malva e juta ${ }^{1}$
}

\section{Screening and dose-response of herbicides to mauve and jute}

\author{
Jefferson Cavalcante Souza²; Ana Marta Lima Nunes Cavalcante²; Gerlândio Suassuna \\ Gonçalves $^{3}$; Sônia Maria Figueiredo Albertino ${ }^{4}$; José Ferreira da Silva ${ }^{4}$
}

\begin{abstract}
Resumo - A produção de fibra de malva (Urena lobata) e de juta (Corchorus capsularis) é demandada pela indústria como matéria prima na produção de telas, cordas, capas de livro entre outras aplicações. O ciclo de produção desta fibra é uma atividade sustentável, pois usa recursos renováveis das várzeas do Rio Solimões no Amazonas. O manejo da cultura aliado ao controle de plantas daninhas são condições para as espécies expressarem o máximo potencial produtivo. O uso de herbicidas seletivos se constitui numa alternativa eficiente e economicamente viável a esta finalidade. O objetivo desta pesquisa foi avaliar a seletividade de herbicidas por meio de screening e a dose-resposta destes as duas espécies produtoras de fibras naturais. Dois experimentos foram conduzidos em casa de vegetação: o primeiro na forma de screening para avaliar herbicidas aplicados em pós-emergência como bentazon, clethodim, fluazifop-p-butil, fomesafen, linuron, oxyfluorfen e sulfentrazone nas doses recomendadas para outras culturas; e o segundo, os herbicidas clethodim e fluazifop-p-butil, que foram seletivos à juta e à malva, em 7 doses. Os herbicidas bentazon, linuron, fomesafen, oxyfluorfen e sulfentrazone causaram morte de todas as plantas, enquanto o clethodim e fluazifop-p-butil foram moderadamente fitotóxicos. As duas espécies foram mais tolerantes ao fluazifop-p-butil que ao clethodim.
\end{abstract}

Palavras-chaves: fibras, seletividade, tolerância

Abstract - The production of mauve (Urena lobata) and jute (Corchorus capsularis) fiber is demanded by the industry as a raw material in the production of canvas, ropes, book covers and other applications. The production cycle of this fiber is a sustainable activity because it uses renewable resources of the floodplains of the Solimões River in the Amazonas state. The crop management combined with weed control are conditions for the species to express the maximum productive potential. The use of selective herbicides constitutes an efficient and economically viable alternative for this purpose. The objective of this research was to evaluate the selectivity of herbicides through screening and dose-response for these two species producers of natural fibers. Two experiments were conducted in a greenhouse: the first, in the form of screening to assess herbicides applied in post-emergence as bentazon, clethodim, fluazifop-p-butyl, fomesafen, linuron, oxyfluorfen, and sulfentrazone at recommended doses for other crops; and the second, the herbicides clethodim and fluazifop-p-butyl that were selective to jute and mauve, in 7 doses. The

\footnotetext{
${ }^{1}$ Recebido para publicação em 11/12/2014 e aceito em 14/08/2015.

${ }^{2}$ Universidade Federal do Amazonas Faculdade de Ciências Agrárias Departamento de Produção Animal e Vegetal. Av. Rodrigo Octavio J. Ramos, 6200. CEP: 697700-000. Manaus- AM. E-mail: <cavalcantebeto@yahoo.com.br>; <anamartacavalcante@yahoo.com.br>.

${ }^{3}$ Docente na Universidade Federal do Amazonas, no Campus de Itacoatiara. CEP: 69100-000. Itacoatiara-AM. E-mail: <gsuassunag@hotmail.com>.

4 Docente na Universidade Federal do Amazonas, no Campus de Manaus. Manaus-AM. E-mail: <sonialbertino@ufam.edu.br>;<jofersil1000@gmail.com>.
} 
herbicides bentazon, linuron, fomesafen, oxyfluorfen, and sulfentrazone caused death of all plants while clethodim and fluazifop-p-butyl were moderately phytotoxic. The two species were more tolerant to fluazifop-p-butyl than to clethodim.

Keywords: fibers, selectivity, tolerance

Nas indústrias, a produção de fibra sintética se sobrepõe à produção de fibra natural, porém esta última tem se destacado devido à forte demanda do mercado por produto sustentável. No Brasil, o estado do Amazonas é o maior produtor de fibras naturais extraídas da malva (Urena lobata L.) e da juta (Corchorus capsularis L.) com 8.700 t, seguido do Pará com $1.140 \mathrm{t}$ (IBGE, 2013). A produção destas fibras tem forte demanda pelas indústrias e a produção é insuficiente para atender o mercado brasileiro pelo produto (Lima et al., 2007).

A oferta parcial da matéria prima ao mercado brasileiro abre espaço para importação de fibras naturais de outros países, principalmente da Índia. Um dos fatores que contribui para a importação é a baixa produtividade da malva e juta devido ao produtor usar a mesma tecnologia da década de 30, quando estas espécies foram introduzidas no Amazonas pelos imigrantes japoneses (Gioia, 2011). A produção da fibra ainda é totalmente manual e ocorre em condições de alta radiação solar, elevada umidade relativa do ar e calor, que contribuem para o baixo rendimento do trabalho.

A importância econômica e social que as culturas da malva e juta representam para o estado do Amazonas requer prioridade $\mathrm{e}$ agilidade no desenvolvimento e implementação de novas técnicas em seu sistema de cultivo. Uma das etapas do sistema de cultivo que necessita de estudo é o manejo das plantas daninhas, devido estas plantas reduzirem a produtividade das culturas e dificultar a colheita e os tratos fitossanitários (Silva et al., 2007). Além disso, estas plantas, geralmente são mais competitivas que as culturas (Ronchi et al., 2007). Assim, o uso de herbicida como inovação tecnológica para estas culturas poderá melhorar o rendimento do trabalho do produtor e a sua margem de lucro com menor custo de produção.
O controle químico das plantas daninhas permite ao produtor, em pouco tempo de serviço, realizar a capina das plantas daninhas e ainda disponibilizar a mão de obra para outra atividade agrícola. No entanto, o uso de herbicidas visando à redução da interferência das plantas daninhas nas culturas de juta e malva é limitado, em decorrência da inexistência de herbicidas registrados para estas espécies. Diante disso, tornam necessárias pesquisas para identificar produtos seletivos a estas espécies.

A seletividade de herbicidas a uma planta pode ser medida pela inibição das características de crescimento, principalmente no acúmulo de matéria seca, dimensão da área foliar e avaliação visual dos danos. Doseresposta é outra importante ferramenta na ciência de plantas daninhas, pois permite a interpretação dos resultados de forma objetiva e possibilita comparação adequada entre tratamentos (Monqueiro et al., 2000). Esta pesquisa teve o objetivo de avaliar a seletividade e dose-resposta de herbicidas pós-emergentes nas culturas de juta e malva.

Esta pesquisa foi conduzida em casa de vegetação com temperatura média de mínima de $22^{\circ} \mathrm{C}$ e máxima de $36{ }^{\circ} \mathrm{C}$, umidade relativa do ar em torno de $80 \%$. Devido à falta de referência, sobre o uso de herbicida nestas culturas, fez dois experimentos, sendo o primeiro tipo screening e o segundo dose-resposta para medir a seletividade dos dois melhores herbicidas às estas culturas.

O delineamento do experimento para screening foi inteiramente casualizado com oito tratamentos e quatro repetições. Os tratamentos foram constituídos da aplicação dos seguintes herbicidas e suas respectivas doses apresentadas em ingrediente ativo: bentazon (720 $\left.\mathrm{g} \mathrm{ha}^{-1}\right)$, clethodim (96 $\mathrm{g} \mathrm{ha}^{-1}$ ), fluazifop-p-butil (188 $\mathrm{g}$ $\left.\mathrm{ha}^{-1}\right)$, fomesafen $\left(238 \mathrm{~g} \mathrm{ha}^{-1}\right)$, linuron $\left(1100 \mathrm{~g} \mathrm{ha}^{-}\right.$ $\left.{ }^{1}\right)$, oxyfluorfen $\left(600 \mathrm{~g} \mathrm{ha}^{-1}\right)$ e sulfentrazone (700 
$\mathrm{g} \mathrm{ha}^{-1}$ ) em mais um tratamento controle, sem aplicação de herbicida. Os herbicidas foram pulverizados aos 39 dias após a semeadura, quando as plantas apresentavam $0,3 \mathrm{~m}$ de altura, aproximadamente, e 15 folhas em média. A aplicação foi no período da manhã com temperatura média de $28^{\circ} \mathrm{C}$ e umidade relativa do ar $88 \%$ com pulverizador costal, pressão constante de $2,5 \mathrm{kgf} \mathrm{cm}^{-2}$ e ponteiras XR 80.03 calibrado para descarga de $160 \mathrm{~L} \mathrm{ha}^{-1}$ de calda com adição de $0,25 \%$ v/v de espalhante adesivo agral à calda de pulverização.

As sementes de malva e de juta foram doadas pelo Instituto de Fomento à Produção de Fibras Vegetais da Amazônia cedidas pelo IFIBRAM-PA e pelo Governo do Amazonas por meio do IDAM. A dormência da semente foi quebrada por imersão em água a temperatura de 60 a $76^{\circ} \mathrm{C}$, durante 12 horas (Figueiredo e Popinigis, 1979). Após esse processo, as sementes foram semeadas em bandejas com substrato Plantmax ${ }^{\odot}$, onde cresceram durante 15 dias e depois transplantadas para sacos de polietileno contendo $3 \mathrm{~kg}$ de solo de várzea retirado da área experimental da Embrapa. A análise da composição química, física e classificação textural do solo foram: $\mathrm{pH}\left(\mathrm{H}_{2} \mathrm{O}\right)$ 5,25; $\mathrm{P}=4 \mathrm{~g} \mathrm{dm}^{-3}, \mathrm{~K}=78 \mathrm{~g} \mathrm{dm}^{-3} ; \mathrm{Ca}, \mathrm{Mg}$, $\mathrm{H}+\mathrm{Al}^{+3}+\mathrm{Sb} 9,8 ; 3,9 ; 3,391,7, \mathrm{em} \mathrm{cmol}_{\mathrm{c}} \mathrm{dm}^{-3}$, recpectivamente. A classe textural do solo utilizado foi areno-argilosa com $24,83 \%$ de areia total, $58,77 \%$ de silte e $16,40 \%$ de argila. Nestes sacos foram transplantadas 4 plântulas de cada espécie estudada e desbastadas, aos 21 dias, deixando apenas 2 plantas por parcela experimental. A irrigação foi diária, sendo calculada para repor a perda de água com base na capacidade de campo do solo.

A avaliação da fitotoxicidade foi realizada aos 20 dias após a aplicação dos tratamentos (DAA), com base na escala EWRC (1964). Após este período, as plantas foram cortadas rente ao solo e separadas as folhas. As características avaliadas foram: área foliar (AF) medida com o equipamento "area metter", marca LI-COR, modelo 3050A e matéria seca total (MST) obtida pela soma do peso da parte aérea e da raiz. A área foliar especifica foi calculada dividindo a área foliar (AF) pelo peso da matéria seca da folha (Cairo et al., 2008).

Os procedimentos e as avaliações utilizados no segundo experimento foram os mesmos do primeiro. Os herbicidas testados foram o clethodim e o fluazifop-p-butil que mostraram maior seletividade às duas culturas. As sete doses de clethodim foram: 0, 12, 24, 48, 96, 144 e 192 g i.a. ha $^{-1}$ e as de fluazifop-p-butil 0, 23, 47, 94, 188, 281 e 375 g i.a.ha ${ }^{-1}$. O delineamento foi o inteiramente casualizado em esquema fatorial $2 \times 2 \times 7$, sendo 2 herbicidas, 2 espécies vegetais e 7 doses de cada produto.

Os dados foram submetidos a análise de variância, com o auxílio do software SAEG 9.1 e quando significativos $(p \leq 0,05)$, fez se a regressão com o auxílio do software Table Curve. Os dados originais foram transformados em $\%$, considerando se a dose zero como $100 \%$ de crescimento, para se obter o valor da dose que inibiu $50 \%$ ( I $\left._{50}\right)$ de cada uma das características avaliadas das espécies produtoras de fibras.

No experimento de screening, os herbicidas clethodim e fluazifop-p-butil causaram intoxicação visual às plantas na forma de clorose nas folhas. Estes herbicidas tiveram comportamentos diferentes para área foliar e a matéria seca total, mas não causaram a morte das duas espécies (Tabela 1). Os demais herbicidas ocasionaram destruição de todos os tecidos da planta, não sendo seletivos à juta e a malva.

Os herbicidas inibidores de Acetil Coenzima-A Carboxilase -ACCase (EC.6.4.1.2) como clethodim e fluazifop-p-butil não deveriam causar injúrias em dicotiledôneas, mas nesta pesquisa reduziram as características de crescimento das duas espécies vegetais produtoras de fibras em relação ao controle (Tabela 1). Isto pode ocorrer em algumas espécies de dicotiledôneas devido esta enzima apresentar, conforme a espécie, três tipos de subunidades: biotina carboxila transportadora, carboxilase de biotina e acetil-coenzima (Wakil et al,1983). 
Os sintomas visuais de injúrias causadas pelos herbicidas às duas espécies de plantas também podem ser explicados pela variabilidade da enzima ACCase nestas plantas de maneira semelhante aos danos em melão, pepino e mandioquinha-salsa (Vidal et al., 2000;
Freitas et al., 2004). Estes danos à malva e a juta, de acordo com Hull e Cobb (1998), podem ocorrer em dicotiledôneas devido os herbicidas inibidores de ACCase poderem expressar efeitos diferenciados, na planta, durante a inibição da $\mathrm{H}^{+}$-ATPase.

Tabela 1 - Fitotoxicidade (\%), área foliar (AF), área foliar específica (AFE) e matéria seca total (MST) de juta e de malva no experimento de screening de herbicidas. Manaus - AM.

\begin{tabular}{|c|c|c|c|c|c|c|c|c|c|}
\hline \multirow{2}{*}{ Tratamentos } & \multirow{2}{*}{$\frac{\text { Doses }}{\text { g i.a.ha }^{-1}}$} & \multicolumn{2}{|c|}{ Fitotoxicidade } & \multicolumn{2}{|c|}{$\operatorname{AF}\left(\mathrm{cm}^{2}\right)$} & \multicolumn{2}{|c|}{$\operatorname{AFE}\left(\mathrm{cm}^{2} \mathrm{~g}^{-1}\right)$} & \multicolumn{2}{|c|}{$\operatorname{MST}(\mathrm{g})$} \\
\hline & & Juta & Malva & Juta & Malva & Juta & Malva & Juta & Malva \\
\hline Controle & 0 & 1 & 1 & $369,6 a$ & $331,1 \mathrm{a}$ & $546,0 \mathrm{a}$ & $392,8 \mathrm{a}$ & $1,2 \mathrm{a}$ & $1,4 \mathrm{a}$ \\
\hline Clethodim & 96 & 2 & 2 & $198,3 b$ & $160,1 \mathrm{~b}$ & $475,6 b$ & $400,1 \mathrm{~b}$ & $0,7 \mathrm{~b}$ & $0,8 b$ \\
\hline Fluazifop-butil & 188 & 5 & 5 & $138,7 \mathrm{c}$ & $53,1 \mathrm{c}$ & $428,3 c$ & $368,7 \mathrm{c}$ & $0,6 \mathrm{c}$ & $0,4 \mathrm{c}$ \\
\hline Bentazon & 720 & 9 & 9 & $0,0 \mathrm{~d}$ & $0,0 \mathrm{~d}$ & $0,0 \mathrm{~d}$ & $0,0 \mathrm{~d}$ & $0,0 \mathrm{~d}$ & $0,0 \mathrm{~d}$ \\
\hline Fomesafen & 238 & 9 & 9 & $0,0 \mathrm{~d}$ & $0,0 \mathrm{~d}$ & $0,0 \mathrm{~d}$ & $0,0 \mathrm{~d}$ & $0,0 \mathrm{~d}$ & $0,0 \mathrm{~d}$ \\
\hline Linuron & 1100 & 9 & 9 & $0,0 \mathrm{~d}$ & $0,0 \mathrm{~d}$ & $0,0 \mathrm{~d}$ & $0,0 \mathrm{~d}$ & $0,0 \mathrm{~d}$ & $0,0 \mathrm{~d}$ \\
\hline Oxyfluorfen & 600 & 9 & 9 & $0,0 \mathrm{~d}$ & $0,0 \mathrm{~d}$ & $0,0 \mathrm{~d}$ & $0,0 \mathrm{~d}$ & $0,0 \mathrm{~d}$ & $0,0 \mathrm{~d}$ \\
\hline Sulfentrazone & 700 & 9 & 9 & $0,0 \mathrm{~d}$ & $0,0 \mathrm{~d}$ & $0,0 \mathrm{~d}$ & $0,0 \mathrm{~d}$ & $0,0 \mathrm{~d}$ & $0,0 \mathrm{~d}$ \\
\hline C.V. $(\%)$ & & & & \multicolumn{2}{|c|}{18,83} & \multicolumn{2}{|c|}{11,807} & \multicolumn{2}{|c|}{4,337} \\
\hline
\end{tabular}

*Médias seguidas de mesma letra nas colunas, não diferem entre si pelo teste de Scott-Knott $(p \leq 0,05)$.

No experimento de dose-resposta, os herbicidas clethodim e fluazifop-p-butil causaram sintomas leves de fitotoxicidade às duas espécies de plantas (Tabela 2). Na juta, o clethodim provocou injúrias visuais na maior dose empregada (144 $\left.\mathrm{g} \mathrm{ha}^{-1}\right)$ como clorose e ${ }^{1}$ ). amarelecimento, enquanto na malva ocorreram clorose e amarelecimento das folhas nas duas maiores doses (144 e $192 \mathrm{~g} \mathrm{ha}^{-1}$ ). Já, o fluazifopp-butil só provocou poucas alterações visuais nas folhas na maior dose empregada $\left(375 \mathrm{~g} \mathrm{ha}^{-}\right.$

Tabela 2- Fitotoxicidade (EWRC, 1964) dos herbicidas clethodim e fluazifop-p-butil aplicados em diferentes doses para juta e malva. Manaus - AM.

\begin{tabular}{cccccc}
\hline Clethodim & \multicolumn{2}{c}{ Fitotoxicidade } & Fluazifop-p-butil & \multicolumn{2}{c}{ Fitotoxicidade } \\
\hline Doses $\left(\mathrm{g} \mathrm{ha}^{-1}\right)$ & Juta & Malva & Doses $\left(\mathrm{g} \mathrm{ha}^{-1}\right)$ & Juta & Malva \\
\hline 0 & 1 & 1 & 0 & 1 & 1 \\
12 & 1 & 1 & 23 & 1 & 1 \\
24 & 1 & 1 & 47 & 1 & 1 \\
48 & 1 & 1 & 94 & 1 & 1 \\
96 & 1 & 1 & 188 & 1 & 1 \\
144 & 1 & 4 & 281 & 3 & 4 \\
192 & 3 & 5 & 375 & &
\end{tabular}

A atividade das doses abaixo da daquela recomendada (188 g i.a. ha ${ }^{-1}$ ) de fluazifop-pbutil promoveu um estímulo positivo nas características de área foliar e de matéria seca total da juta. Porém, a dose de $375 \mathrm{~g} \mathrm{ha}^{-1}$ causou decréscimo nos valores para estas características, principalmente da matéria seca total, que teve redução na ordem de $10 \%$ em relação ao controle (Figura 1).
Em relação à malva, todas as características avaliadas foram reduzidas pelo fluazifop-p-butil nas doses acima da recomendada (188 g i.a. ha ${ }^{-1}$ ). A maior dose, $375 \mathrm{~g} \mathrm{ha}^{-1}$ reduziu a área foliar em $55 \%$ e a matéria seca total em $63 \%$, aproximadamente, em relação ao tratamento controle.

A redução no acúmulo de matéria seca, segundo Thill (2000), deve-se ao mecanismo de 
ação primário do fluazifop-p-butil , que inibe a síntese de ácidos graxos, por meio da inibição da enzima ACCase. Tal mecanismo age diretamente nos pontos de crescimento da planta, que apresentam intensa divisão e elongação celular. Dessa maneira, impede a formação de novas células e o crescimento vegetal.

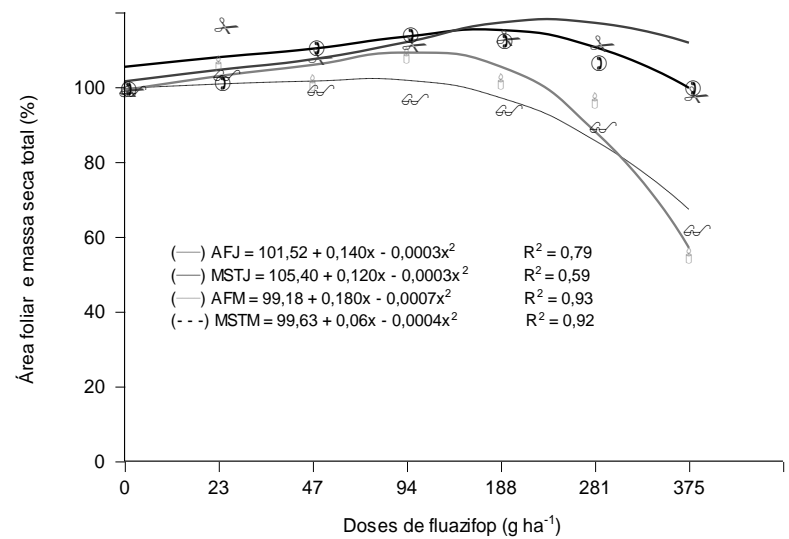

Figura 1. Dose-resposta do herbicida fluazifopp-butil em plantas de juta e malva. Área foliar da juta (AFJ), matéria seca total da juta (MSTJ), área foliar da malva (AFM) e matéria seca total da malva (MSTM). Manaus - AM.

Apesar do incremento das doses, acima da recomendada (188 g i.a. ha ${ }^{-1}$ ) ter provocado lesões foliares, a área foliar e a massa seca total das plantas de juta não foram afetadas negativamente pelo fluazifop-p-butil.

O clethodim reduziu a matéria seca e a área foliar da malva e a juta mostrou-se mais tolerante ao herbicida que a malva (Figura 2). A dose de clethodim que inibiu $50 \%$ da área foliar da malva foi $190 \mathrm{~g} \mathrm{ha}^{-1}$. Isso demonstra que, para produzir danos com mesma intensidade daquela produzida pelo clethodim, o fluazifopp-butil precisaria ser aplicado em dose bem acima da recomendada (96 g i.a. ha ${ }^{-1}$ ) para outras culturas. Este comportamento pode ser explicado pela maior tolerância de plantas de malva ao herbicida fluazifop-p-butil em relação ao clethodim e por características de cada herbicida. Ambos são absorvidos pelas folhas e apresentam o mesmo mecanismo de inibir a atividade da ACCase, tendo como consequência a inibição da divisão celular, da formação de cloroplastos e diminuição da respiração e do crescimento, porém, a translocação destes produtos na planta é diferenciada (Christopher e Holtum, 1998).

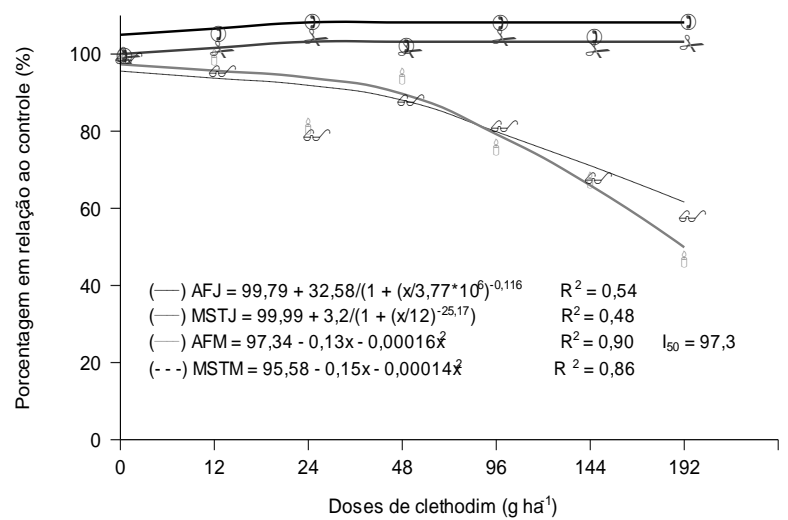

Figura 2. Doses-resposta de juta e malva em relação ao herbicida clethodim. Área foliar da juta (AFJ), matéria seca total da juta (MSTJ), área foliar da malva (AFM) e matéria seca total da malva (MSTM). Manaus - AM.

Apesar dos danos terem sido maiores nas folhas, o acúmulo de matéria seca do caule e das raízes também foi afetado, ocasionando redução no valor da matéria seca total. Na presença do clethodim, o acúmulo de matéria seca total tanto da juta como da malva, foi no máximo $65 \%$ e $55 \%$ respectivamente daquele apresentado pelo controle.

\section{Conclusões}

Os herbicidas bentazon, fomesafen, linuron, oxyfluorfen e sulfentrazone causaram a morte das plantas de malva e juta, enquanto clethodim e fluazifop-p-butil foram seletivos a essas culturas.

\section{Referências}

CAIRO, P.A.R.; OLIVEIRA, L.E.M.; MESQUITA, A.C. Análise de crescimento de 
plantas. Vitória da Conquista: Edições UESB, p. 71, 2008.

CHRISTOPHER, J.T.; HOLTUM, J.A.M. The dicotyledonous species Erodium moschatum (L) L'Hér. ex. Aiton is sensitive to haloxyfop herbicide due to herbicide-sensitive acetylcoenzyme A carboxylase. Planta, v.207, n.2, p.275-279, 1998.

EUROPEAN WEED RESEARCH COUNCIL. Report of 3rd and 4th meetings of EWRC. Committee of Methods in Weed Research. Weed Research, v.4, p.88, 1964.

FIGUEIREDO, F.J.C.; POPINIGIS, E. Superação da dormência de sementes de malva (Urena lobata L.). BelSm, CPATU, 1979. 18p. (Comunicado Técnico, 21).

FREITAS, R.S. et al. Seletividade de herbicidas aplicados em pós-emergência para a cultura da mandioquinha-salsa. Planta Daninha, v.22, n.1, p.159-165, 2004.

GIOIA, D.L.A. Cultivo de juta no AM é resultado de trabalho da colônia japonesa, G1, São Paulo, dez. 2011. Seção Economia. Disponível

<http://g1.globo.com/economia/agronegocios/v ida-rural/noticia/2011/12/cultivo-de-juta-naam-e-resultado-de-trabalho-da-coloniajaponesa.html>. Acesso em 21 jan. 2011.

HULL, M.R.; COBB, A.H. An investigation of herbicide interaction with the H+-ATPase activity of plant plasma membranes. Pest Science, v.53, n.2, p.155-164, 1998.

IBGE - Instituto Brasileiro de Geografia e Estatística Disponível em: <http://www.sidra.ibge.gov.br/bda/agric/defaul t. asp?t=2\&z=t\&o=11\&u1=33\&u3=1\&u4=1\&u $5=1 \& u 6=1 \& u 2=13>$. Acessado em 9 de agosto de 2015.

LIMA, H.; TEIXEIRA, W. Amazônicas modos de vida e uso dos recursos naturais: os solos da paisagem da várzea com ênfase no trecho entre Coari e Manaus. 1.ed. Manaus: EDUA, 2007. $36 \mathrm{p}$.
MONQUEIRO, P.A.; CHRISTOFOLLETI, P.J.; DIAS, C.T.S. Resistência de plantas daninhas aos herbicidas inibidores da ALS na cultura da soja (Glycine max). Planta Daninha, v.18, n.3, p.419-423, 2000.

RONCHI, C.P. et al. Growth and nutrient concentration in coffee root system under weed species competition. Planta Daninha, v.25, n.4, p.679-687, 2007.

SILVA, A.A.; FERREIRA, F.A.; FERREIRA, L.R. Herbicidas: classificação e mecanismos de ação. In: SILVA, A.A.; SILVA, J.F. (Editores).

Tópicos em Manejo de Plantas Daninhas. Viçosa, MG: UFV, p. 83-148, 2007.

VIDAL, R.A. et al. Seletividade do herbicida fluazifop-p-butil para cucurbitáceas. Planta Daninha, v.18, n.3, p.413-417, 2000.

WAKIL, S.J.; STOOPS, J.K; JOSHI, V.C. Fatty acid synthesis and its regulation. Annual Reviews of Biochemistry, v.52, p.537-579. 1983. 